\section{In vitro beneficial effects of a flax extract on papillary fibroblasts define it as an anti-aging candidate}

\author{
PAGEON Hervé*, ZUCCHI Hélène, RICOIS Sylvie, BASTIEN \\ Philippe and ASSELINEAU Daniel
}

L’Oréal Research and Innovation, Aulnay-sous-bois, France

\section{Abstract}

Objective: During aging, skin undergoes structural, cellular and molecular changes, which not only alter skin mechanical properties but also biological and physiological functions. Structurally the epidermis becomes thinner, the dermal epidermal junction flattens and the extracellular matrix component of the dermis is disorganized and degraded. The dermis is composed of two compartments: The Reticular dermis is the deepest and thickest part while the upper layer, the papillary dermis, which is much thinner and is in close contact with epidermis, plays an important role in the structure and function of the skin. We have recently shown that the papillary dermis was preferentially affected by skin aging because the activity of fibroblasts in this region was especially altered as a function of age. The purpose of this study was to investigate the capacity of a flax extract as anti-aging component.

Method: We investigated the capacity of a flax extract to stimulate or restore the activity of papillary fibroblasts from young and old donors in cultured monolayers and in reconstructed skin. Several biological markers of extracellular matrix homeostasis and mechanical properties were investigated.

Results: The tested flax extract seemed to improve parameters known to change with age: I/ In monolayers after treatment the number of aged fibroblasts increased II/ In reconstructed skin the flax extract appears to positively regulate some biological activities; particularly in aged fibroblasts where the deposition of laminin 5, fibrillin 1, procollagen I were increased in the dermis and the secretion of specific soluble factors like MMP1, MMP3 and KGF were regulated to levels similar to those observed in young fibroblasts III/ Mechanical properties were improved particularly for elastics parameters (R5, R2 and R7).

Conclusion: The flax extract is a promising anti-aging compound. The treatment of aged papillary fibroblasts resulted in a return to a younger-like profile for some of the studied parameters.
More Information

*Address for Correspondence:

PAGEON Hervé, L'Oréal, Research \& Innovation, 1 Avenue Eugène Schueller, 93600 Aulnay-sousbois, France, Tel: 33-1-48-68-92-42; Email: herve.pageon@rd.loreal.com

Submitted: February 10, 2021

Approved: May 04, 2021

Published: May 05, 2021

How to cite this article: PAGEON H, ZUCCHI H, RICOIS S, BASTIEN P, ASSELINEAU D. In vitro beneficial effects of a flax extract on papillary fibroblasts define it as an anti-aging candidate. Arch Biotechnol Biomed. 2021; 5: 032-040.

DOI: 10.29328/journal.abb.1001026

Copyright: @ 2021 PAGEON H, et al. This is an open access article distributed under the Creative Commons Attribution License, which permits unrestricted use, distribution, and reproduction in any medium, provided the original work is properly cited.

Keywords: Cell culture; Skin physiology; Flaxextract; Skin aging; Fibroblasts; Mechanical properties

(A) Check for updates

(9) OPEN ACCESS

\section{Introduction}

During aging skin undergoes structural, cellular and molecular changes, which not only alter skin mechanical properties but also biological and physiological functions. Many modifications appearing in characteristic of skin aging, which have been reported in the literature, result from intrinsic but also extrinsic aging. Overall, a production of ROS was observed that leads to cell oxidation, the stimulation of proinflammatory cytokines, which generate an inflammatory microenvironment, a modification of collagen metabolism due to decrease in its synthesis and an increase in its degradation by the induction of MMPs activities. More specifically, in the case of extrinsic aging and notably through exposure to UV, the metabolism of the elastic network was altered due to the induction of tropoelastin mRNA level and the abnormal accumulation of elastin in the dermis [1,2]. Characteristic changes in skin structure due to age are commonly observed by histological staining: a thinning of the epidermal thickness, the dermal epidermal junction flattens and the deepest layer of the dermis named the reticular dermis, which is thickest part, is disorganized and degraded (Figure 1) leading to a loss of elastic properties of the skin. Nevertheless, the papillary dermis, which is much thinner and in closer contact with the epidermis, also plays an important role in the structure and function of the skin through its constituents [3-5] and cells [6] that compose it. Especially, we have shown that papillary dermis was affected by skin aging due to an alteration of 
fibroblast activity as a function of age. For example, aged papillary fibroblasts increased their secretion of soluble molecules such as MMPs involved in extracellular matrix degradation [7]. Additionally, since the papillary dermis is highly exposed to extrinsic aging, molecules that prevent aging of this specific part of the skin dermis are sought after. Previously, it has been reported that using compounds such as retinoic acid [8] or vitamin $\mathrm{C}$ [9] may improve the properties of skin and counter the effects of age. These compounds have been tested in 2D culture but also in reconstructed skin models [10-12]. We have shown recently that rhamnose stimulated the activity of papillary fibroblasts from young or old donors in in vitro reconstructed skin models and that this molecule represents a potential anti-aging molecule [13]. It seems important to develop new active ingredients to improve the deficient properties of these papillary fibroblasts in aged skin. Thus the purpose of our study was to investigate the effect of a flax extract to stimulate or restore the biological activity of papillary fibroblasts from young or old donors in cultured monolayers and in reconstructed skin. We have also explored if the regulation of papillary fibroblast activity can stimulate the cell to modify its extracellular environment and change the mechanical properties of the dermis in in vitro skin models. We conclude that the flax extract may represent a potential anti-aging molecule by improving both the biological and mechanical properties of skin in vitro.

\section{Materials and methods}

\section{Chemicals}

Flax extract (Linactyl ${ }^{\circledR}$ ) was from Silab (Saint-Viance, France).

\section{Fibroblast isolation}

Samples of human breast skin were obtained from plastic surgery of two adult European-Caucasian women (young: 23 years-old and old: 63 years-old) and this anatomical site is considered to be sun non-exposed. Informed consent was obtained before tissue collection according to Helsinki declaration guide lines. Papillary fibroblasts were isolated from these skin samples using the technique described previously by Mine, et al. Briefly, using a keratome (Aesculap, Braun, Boulogne Billancourt, France), human skin samples were cut to $0.3 \mathrm{~mm}$ in depth from the surface to separate both epidermis and papillary dermis from the rest of the skin. Papillary dermal part was obtained after separation with epidermis and was used to make explants cultivated in Petri dishes in order to isolate the corresponding spreading papillary fibroblasts [7].

\section{Fibroblasts in monolayer}

The papillary fibroblasts isolated from the human breast skin of a young (23 yo) and an elderly donor (63 yo) were seeded on plastic (density 30,000 cells per $\mathrm{cm}^{2}$ in $60 \mathrm{~mm}$ dish) in the presence of Modified Eagle's medium (MEM, Gibco,
Invitrogen, Carlsbad, CA, USA) supplemented with $10 \%$ foetal bovine serum (D. Deutscher, Brumath, France), penicillinstreptomycin (20 U mL_1) (Biochrom, Cambridge Ldt, UK), $2 \mathrm{mM}$ L-glutamine, Sodium pyruvate and Non Essential Amino acids (Gibco, Invitrogen Carlsbad, CA, USA) and maintained at $37^{\circ} \mathrm{C}$ in a $5 \% \mathrm{CO} 2$ and $90 \%$ humidity atmosphere. After $48 \mathrm{hrs}$ of culture, the medium was changed with medium containing Flax extract $1 \%$. Controls were prepared with medium without Flax extract. After $48 \mathrm{hrs}$ of culture, the cells were counted ( $n=3$ per conditions) and the cells lysed ( $n=3$ per conditions) for qRT-PCR.

\section{Skin reconstructed in vitro}

Keratinocytes used for the epidermis reconstruction were obtained from a 33-year-old single donor skin sample after breast reduction. These keratinocytes were cultivated on feeder-layer with 3T3 murine fibroblasts [14]. After amplification keratinocytes were kept frozen in liquid nitrogen until the use for skin in-vitro reconstruction experiments.

The in vitro skin reconstruction procedure has been previously described in details $[15,16]$. Briefly, dermal equivalents were prepared using human papillary dermal fibroblasts embedded into collagen gels. After contraction, epidermis was obtained by seeding human epidermal keratinocytes on the dermal equivalent. The cultures were kept submerged for one week and then raised at the air-liquid interface for one week.

Flax extract at $1 \%$ was added to the culture medium for each medium change from the last change before the cultures were raised at the air-liquid interface and during the course of air-liquid interface culture (a total of 3 stimulations of flax extract per samples during the culture). Three control or flax extract-treated reconstructed skin samples were prepared, using papillary fibroblasts in the dermal part previously isolated from young and old skin samples.

Analysis of the reconstructed skin was carried out by histology, immunochemistry, Elisa assay (analysis of the medium) and mechanical measurement (Rheometer).

\section{Histology}

Reconstructed skin ( $n=3$ per conditions) were fixed in $4 \%$ phosphate-buffered formaldehyde and embedded in paraffin. $5 \mu \mathrm{m}$ tissue sections were mounted onto slides and stained with hematoxylin-eosin-safron (HES) according to standard procedures. After histological coloration, sections were examined under a Leica DMRB microscope (Leica, Microsystems, Wetzlar, Germany).

\section{Immunohistochemistry}

Samples of reconstructed skin ( $n=3$ per conditions) were embedded in Tissue Tek OCT compound (Miles Inc., Elkart, IN, USA), frozen in liquid nitrogen and cut into $5 \mu \mathrm{m}$ thick sections (cryostat, CM3050 S, Leica, Microsystems, Wetzlar, 
Germany). Procollagen I and Laminin 5 were stained using human monoclonal antibodies from Chemicon (Temecula, CA, USA), Fibrillin 1 was stained with human antibody from SouthernBiotech (Birmingham, AL, USA). FITC conjugates were used to detect primary antibodies (Molecular probes, Eugene, OR, USA). Propidium iodure was used at the end of immunostaining to observe nucleus of cells. Stained tissue sections were examined and imaged using a Leica DMRB fluorescence microscope.

Quantitative immunohistochemical evaluations were performed by using software IMAGE J. Three measure of integrated emission per samples were performed (AU: intensity of fluorescence per surface units $-\mu \mathrm{m}^{2}$ ).

\section{ELISA assays}

ELISA were performed on culture medium of reconstructed skin ( $n=3$ per conditions) for Matrix-Metalloproteinase type 1 (MMP-1) (Biotrak ${ }^{\mathrm{TM}}$ RPN2610, Amersham, Buckinghamshire, UK), Matrix-Metalloproteinase type 3 (MMP-3) (Quantikine DMP300, R\&D Systems, Minneapolis, MN, USA) and Keratinocyte Growth factor (Quantikine DKG00, R\&D Systems, Minneapolis, MN, USA).

\section{$\mathrm{RTq}-\mathrm{PCR}$}

Reconstructed skin samples ( $n=3$ per conditions) were rinsed in phosphate buffered saline Dulbecco's without calcium and magnesium (Gibco BRL, Cergy Pontoise, France). Immediately, epidermis and dermal equivalent were separated using fine forceps and directly frozen. Only the dermal part was considered for this analysis. The different steps for molecular biology analysis (RNA extraction, Reverse Transcription, Quantitative reverse transcriptase-PCR) were performed by BioAlternatives Society (Gencay, France). Level expression of mRNA for Fibrillin 1 - FBN1 (NM_000138), Fibulin 5 - FBLN5 (NM_006329), Collagen 16 alpha 1 subunit precursor - COL16A1 (NM_001856), Collagen 6 alpha 1 subunit - COL6A1 (NM_001848), Collagen 7 alpha 1 subunit COL7A1 (NM_000094), Versican core protein precursor CSPG2 (NM_004385), Decorin - DCN (M14219), Biglycan - BGN (NM_001711), Lumican - LUM (NM_002345), Collagen 12 alpha 1 subunit precursor - COL12A1 (NM_080645) were analyzed from the samples. Analysis of gene expression was normalized with 2 housekeeping genes: Liver glyceraldehyde 3-phosphate dehydrogenase - G3PDH (NM_002046) and Ribosomal protein L13A - RPL13A (NM_012423)).

\section{Reverse transcription}

Total cellular RNA was isolated from the dermal part with the Trizol Reagent (Sigma-Aldrich)/Chloroform mixture by isopropanol precipitation and extensively treated with DNase I (Kit DNase-free, Ambion). RNA quantity and quality were analyzed using Bioanalyser (Agilent Tech.). mRNA was reverse-transcribed using the primer oligo(dT) and Supercript II enzyme (Gibco). cDNA was quantified and adjusted using Nanovue (GE Healthcare).

\section{Quantitative PCR}

cDNAs were subsequently analyzed in duplicate by quantitative real-time PCR using the LightCycler system (Roche Diagnostics, Meylan, France) according to the manufacturer's instruction. For each sample, $2.5 \mu \mathrm{L}$ of cDNA were mixed with primers and enzymatic kit (Roche) containing taq DNA polymerase enzyme, SYBR Green I marker and $\mathrm{MgCl}$. Housekeeping mRNAs were quantified in each sample and used for normalization using Rest software.

\section{Biomechanics measurement with Rheometer}

Mechanical measurements were made on the dermal part (punched at diameter $=1 \mathrm{~cm}$ before analysis) of the reconstructed skin sample after separation from the epidermis using forceps. The dermal parts of the samples were prepared with the papillary fibroblasts isolated from the young donor ( $n=5$ per conditions). The mechanical measurement of the samples was carried out with the Malvern Rheometer (Model KNX2210, Malvern Instruments, Malvern, UK).

A protocol of viscoelastic properties in shear was used, followed by the creep recovery protocol in shear (application of a shear stress of $10 \mathrm{~Pa}$ for $300 \mathrm{~s}$ followed by recovery at 0 Pa for $500 \mathrm{~s}$ ). Several parameters can be measured in order to characterize the mechanical property of samples I/ for properties in shear: the rigidity (module $\mathrm{G}^{\prime}$ in $\mathrm{Pa}$ ) and the viscoelasticity (phase angle $\delta$ in degrees). II/ for the recovery test: the parameters in recovery R2, R5 and R7 which translates the lack of elasticity (no return, $\mathrm{R}=0$ ) or a perfect elasticity $(R=1)$. These parameters are calculated from the different parts of the deformation curve (below) as a function of the time recovery.

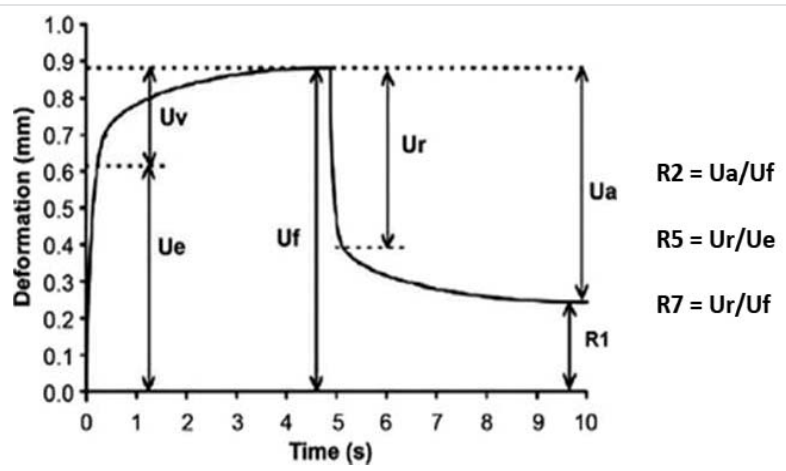

These elasticity parameters characterizing the ability of the material to recover its initial shape.

\section{Statistical analysis}

Means and standard errors of the means were determined from three (immunochemistry and ELISA) or five samples (Rheometer) for each culture conditions represented in box plots. An analysis of variance (ANOVA) was performed to assess whether there are differences between the conditions. When significant, ANOVA was followed by adjusted post-hoc Tukey-Kramer tests for pairwise comparisons. The two-sided 
significance level was set at $5 \%$. Data with $p$ values $<0.05$ were considered as significant.

\section{Results}

\section{Flax extract improved the fibroblasts' biology}

Aging skin exhibits well-known histological features such as decreased epidermal thickness, a flattened dermoepidermal junction, and atrophied dermis. These different features were found in young (Figure 1A) and aged (Figure 1B) skin samples from which papillary fibroblasts, which were used in the experiments in this study, were isolated from the papillary dermis compartment. In previous studies we have already shown that the proliferation of papillary fibroblasts was reduced with aging [7]. In this study at the end of the culture, the number of fibroblasts between young $(\mathrm{Y})$ and aged (0) decreases by $-46 \%(p<0.05)$. Treatment with flax extract seems to increase the proliferation of fibroblasts, particularly in the elderly donor (trend at $+15 \%, p=0.09$ ) (Figure 1C). The expression of several genes coding for components of the extracellular matrix were analyzed. In figure 2 the histograms present the fold change of the selected mRNAs after treatment with the flax extract in comparison to the untreated control for young (Figure 2A) and aged (Figure 2B) fibroblasts. In young fibroblasts, after treatment with flax extract, mRNA expression for type VI, XII, XVI collagens significantly increased with a fold change $\mathrm{Fc}>2(p<0.05)$ and the expression of proteoglycans biglycan, lumican mRNA slightly increased ( $\mathrm{Fc}>1.2 ; p<0.05)$. In fibroblasts from the old donor, a slight significant increase (Fc $>1.2 ; p<0.05)$ in Fibrillin1, Versican (CSPG2) and type XII collagen $(\mathrm{Fc}>1.8 ; p<0.05)$ mRNA expression wasobserved.

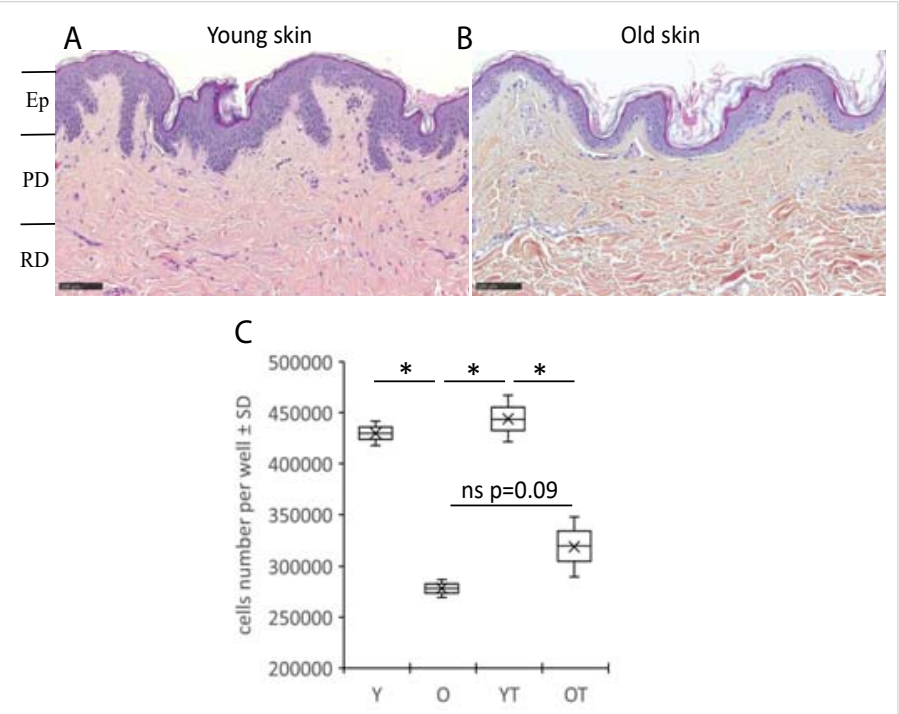

Figure 1: Histological human skin sections from a young donor of 23 yo (A) and an old donor of 63 yo (B) stained with Haematoxylin Eosin Saffron (HES). Ep: Epidermis; PD: Papillary Dermis; RD: Reticular Dermis. (Bar $=100 \mu \mathrm{m})$. Number of cells $(C)$ from papillary dermal part of both donors was quantified. Fibroblasts isolated from a young $(\mathrm{Y})$ or old $(\mathrm{O})$ donor are cultured in the presence or absence of the flax extract (YT and OT). Boxplots ( $n=2$ for samples not treated and $n=3$ for treated samples) \pm SD are reported. Statistics: analysis of variance (ANOVA) adjusted post-hoc Tukey-Kramer tests for pairwise comparisons $\left({ }^{*} p<0.05\right)$.
Flax extract improves the quality of the dermis in in-vitro reconstructed skin, especially in the aged fibroblasts-populated dermal compartement

The tridimensional reconstructions of the skin made with either young or old fibroblasts were well achieved in the presence of flax extract added to the culture medium as shownby the macroscopic pictures (Figure 3A-D). Indeed, the overall architecture of epidermis was very similar to the control (Figure $3 \mathrm{E}-\mathrm{H}$ ). Immunostaining of the basement membrane zone proteins was also performed. Laminin 5 staining was characterized by a thin continuous signal at the dermal-epidermal junction zone and seems to decrease in models with old fibroblasts as compared to young fibroblasts (Figure $3 \mathrm{~J}$ versus I, respectively). In the presence of flax extract, a similar pattern was observed but the staining of Laminin 5 seems to be stronger when compared to untreated conditions (Figure $3 \mathrm{~L}, \mathrm{~K}$ ). This increase was specifically observed for reconstructed skins made with old fibroblasts ( $+64 \%$ after treatment) as compared to those made with young fibroblasts as indicated after signal quantification (Figure 3U) suggesting an increase in synthesis and deposition of this basement membrane component. In both reconstructed skins made with either young or old fibroblasts, a diffuse dermal staining of fibrillin 1 and procollagen I was

A

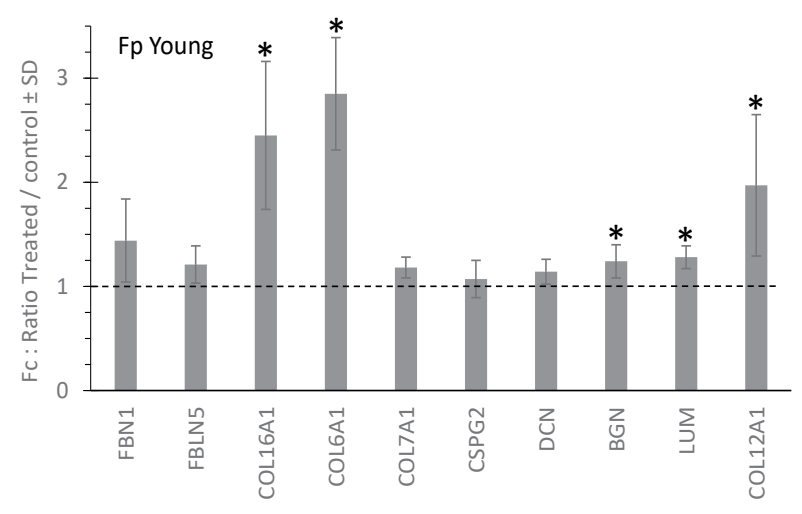

B

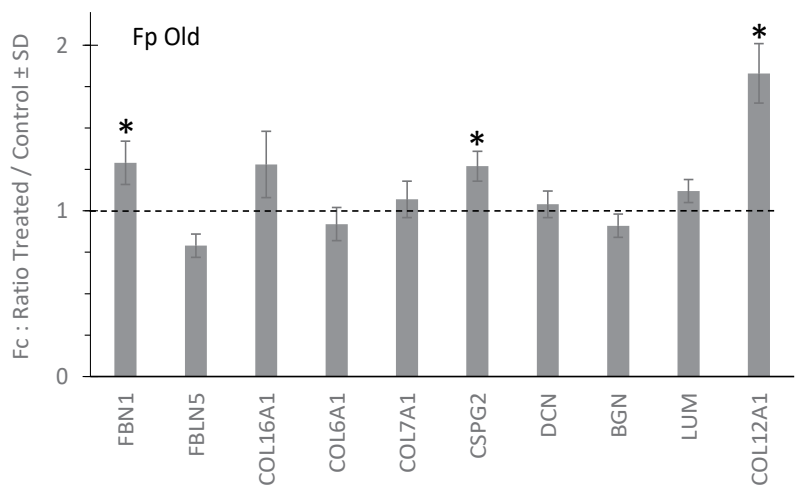

Figure 2: Extracellular matrix mRNA expression measured in young $(A)$ and old $(B)$ fibroblasts cultivated in monolayers. The histograms represent the fold change after 48 h-treatment with flax extract at $1 \%$ compared to the untreated control. mRNA levels were quantified using qPCR at the end of culture. Statistics: analysis of variance (ANOVA) adjusted post-hoc Tukey-Kramer tests for pairwise comparisons $\left({ }^{*} p<0.05\right)$. 


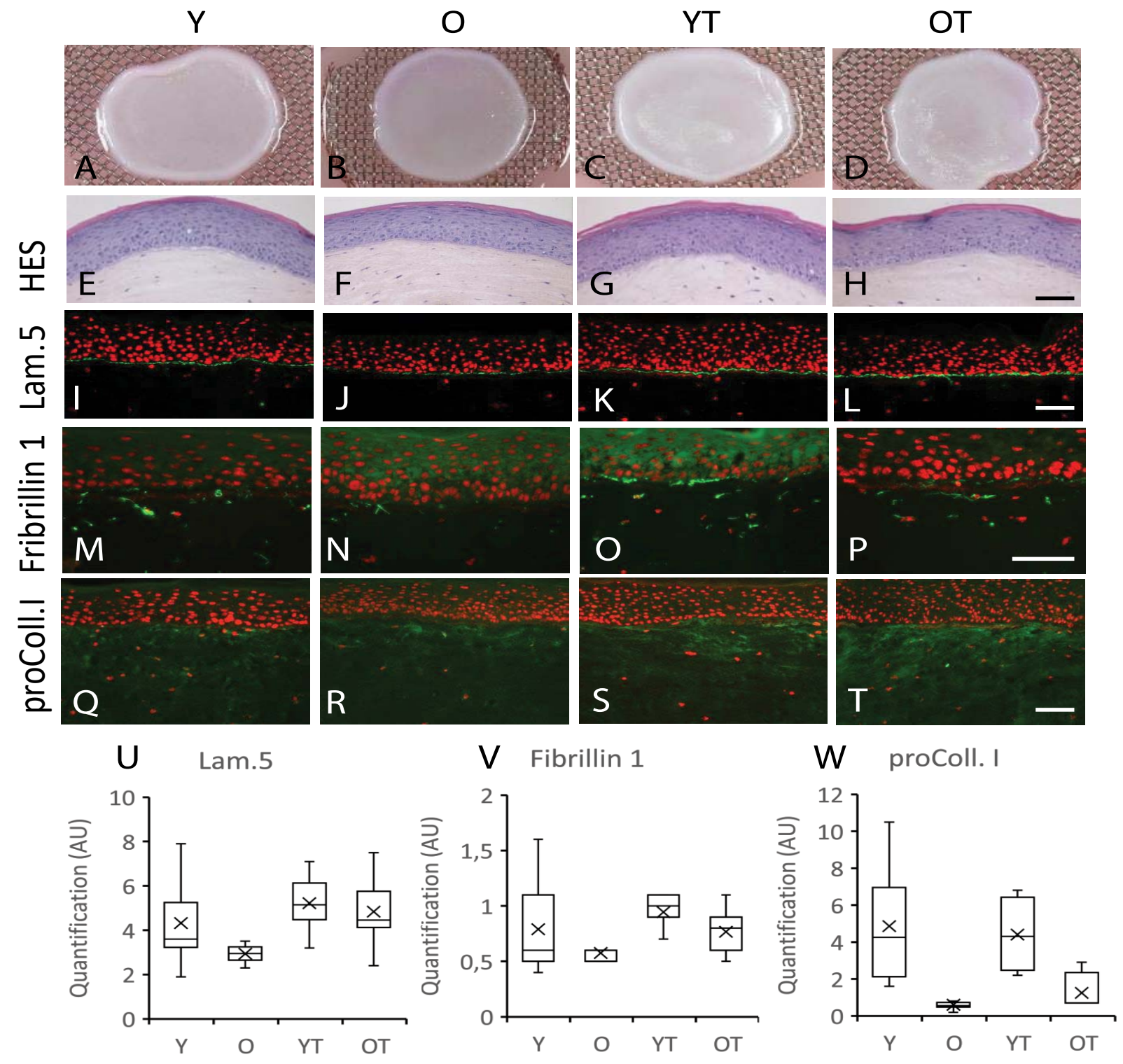

Figure 3: Macroscopic appearance (A-D), HES histological staining (E-H), laminin $5(I, J)$, fibrillin $1(M-P)$ and procollagen I (Q-T) immunostainings of skin reconstructed in vitro using fibroblasts in the dermis part isolated from young or old donors treated (respectively YT or OT) or not treated (respectively $Y$ or $\mathrm{O}$ ) with the flax extract $(\mathrm{Bar}=50 \mu \mathrm{m})$. Box plot represents the Detection of the fluorescent labeling was quantified for laminin $5(\mathrm{U})$, fibrillin $1(\mathrm{~V})$ and procollagen I (W).

observed throughout the dermis. These depositions were lower for models with old fibroblasts when compared to those with young fibroblasts (Figure $3 \mathrm{M}, \mathrm{N}, \mathrm{Q}, \mathrm{R}$, respectively) and confirmed by quantification (Figure $3 \mathrm{~V}, \mathrm{~W}$ ). In the presence of flax extract, the dermal staining was increased specifically for the oldest fibroblasts (Figure $3 \mathrm{P}$ versus $\mathrm{N}$ and $\mathrm{T}$ versus $\mathrm{R}$ ) for both fibrillin 1 and procollagen I while no modification seemed to be observed with young fibroblasts (Figure $3 \mathrm{M}-\mathrm{O}$, Q-S). The immunofluorescent signal increased in dermal compartment by $+34 \%$ and $+108 \%$, respectively, for fibrillin 1 and procollagen I in models with the oldest fibroblasts (Figure $3 \mathrm{~V}, \mathrm{~W})$. Taken together, our results indicated that synthesis of several extracellular matrix molecules were increased in flax extract-treated reconstructed skin made with old fibroblasts.
Flax extract regulates the secretion of soluble factors in the medium of reconstructed skin

Since the soluble factors released in the matrix can influence the quality of the dermis and indirectly the global homeostasis of the skin, we studied in particular those factors in relation to the degradation of the dermal matrix such as the metalloproteinases MMP1 and MMP3, which are specifically expressed by fibroblasts. MMP1 and MMP3 were increased in the medium of the reconstructed skin made with fibroblasts from elderly donors compared to those from young donors (respectively for MMP1: $88+/-4$ vs. $74+/-5 \mathrm{ng} / \mathrm{ml}, p<0.05$ and MMP3: $133+/-11$ vs. $19+/-1 \mathrm{ng} / \mathrm{ml}, p<0.05$ ) (Figure $4 \mathrm{~A}, \mathrm{~B}$ ). Treatment with flax extract resulted in a significant reduction in MMP1 and MMP3 secretion in reconstructed skin made 
A

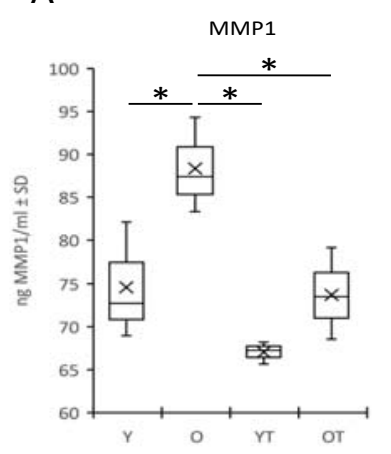

C

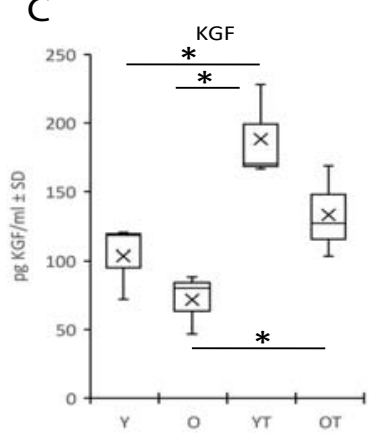

B

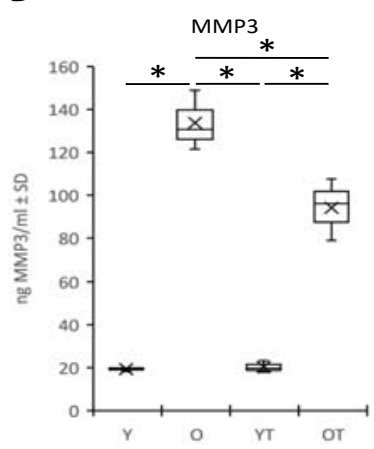

Figure 4: Secretion of matrix metalloproteinase type 1 (MMP1) (A) and type 3 (MMP3) (B), Keratinocyte Growth Factor (KGF) (C). ELISA assays were performed on the culture medium of reconstructed skin made with fibroblasts isolated from young or old donors in the presence (respectively YT or OT) or absence (respectively $\mathrm{Y}$ or $\mathrm{O}$ ) of Flax extract. Statistics: analysis of variance (ANOVA) adjusted post-hoc Tukey-Kramer tests for pairwise comparisons $\left({ }^{*} p<0.05\right)$.

\section{A}
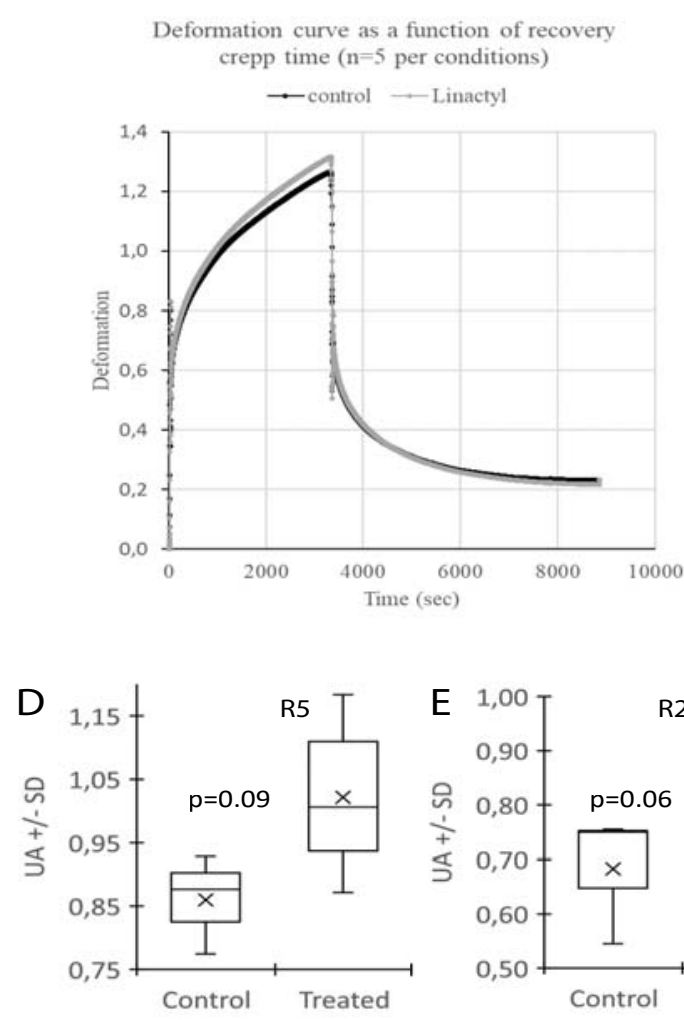

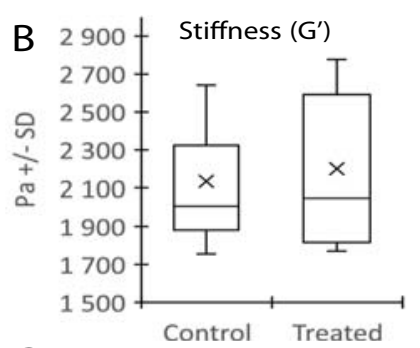

C
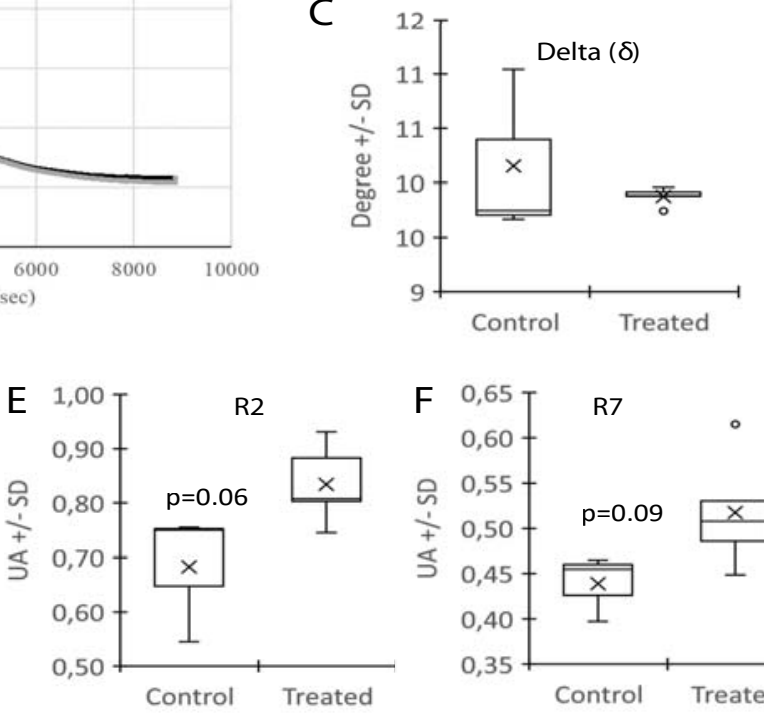

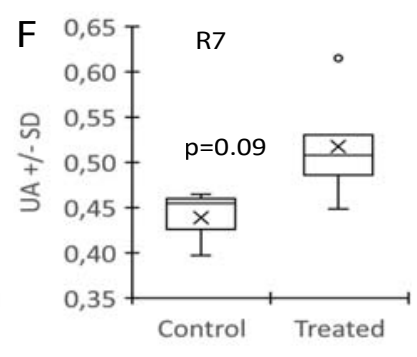

Figure 5: Mechanical properties of the dermal part of reconstructed skin with (Treated) or without (Control) Flax extract, measured with the rheometer. Deformation curve (A), Stiffness $G^{\prime}(B)$, Delta - $\delta(C)$ and elastic parameters R5 (D), R2 (E) and R7 (F) between the both conditions were represented. Statistics: analysis of variance (ANOVA) adjusted post-hoc Tukey-Kramer tests for pairwise comparisons $\left({ }^{*} p<0.05\right)$. 
with old fibroblasts $(-20 \%$ and $-43 \%$ respectively, $p<0.05)$ as compared to samples without treatment. A difference in KGF production was also observed (trend between models with young and old fibroblasts, respectively $103+/-22$ and $71+/-$ $17 \mathrm{pg} / \mathrm{ml}$ ) (Figure $4 \mathrm{C}$ ). Treatment with flax extract increased the production of KGF for both fibroblasts $(p<0.05),+45 \%$ for young and $+47 \%$ for the elderly fibroblasts models as compared with their respective control samples.

\section{Flax extract improves the mechanical properties of reconstructed skin in vitro}

Since, aging of the skin is characterized by a reduction in biological but also physical properties, the effect of flax extract on mechanical parameters were investigated in vitro. A shift on the deformation curve between the samples in the presence or absence of flax extract was observed (Figure 5A). The stiffness $\left(G^{\prime}\right)$ and viscoelasticity $(\delta)$ were not altered (Figure 5 B,C). The elastic parameters R5, R2, R7 (Figure 5D-F) were increased (respectively $+19 \%, p=0.09 ;+22 \%, p=0.06$; and $+18 \%$, $p=0.09$ ) in trend after the treatment which indicates that the material (equivalent dermis) may increase its elastic property and may favor a return to its initial state after deformation thus reflecting its physical elastic capacity. These promising results need to confirmed with other donors and may indicate that mechanical properties improve in dermal compartment after treatment with Flax extract.

\section{Discussion}

In this study we investigated the effect of a flax extract on 2D and 3D skin cultures to measure its influence on changes in biological but also mechanical properties. We also focused on the effect of flax extract on a subpopulation of fibroblasts located in the papillary dermis close to the epidermis: the papillary fibroblasts. The biological characteristics of this dermal population are specifically altered with age [7] because of intrinsic aging and extrinsic factors. Its proximity to the external surface increase its exposure to deleterious external effects like UVA [17].

Skin aging affects the different layers of the dermis and their components: cells and the matrix. In monolayer cultures, the proliferation of fibroblasts decreases with age, and the expression of mRNA coding for the components of the matrix or its degradation, the expression of cytokines, proteins and enzymes have been described as being modulated with cell aging $[7,18]$. The treatment of aged papillary fibroblasts with our flax extract seemed to increase the proliferation of fibroblasts and regulated the secretion of proteins. Among these, MMPs are a family of ubiquitous endopeptidases that can degrade ECM proteins [19]. Previous studies have demonstrated that the levels of MMPs like MMP3 are increased in aged papillary fibroblasts [7] and human skin [20]. Using our model of reconstructed skin containing papillary fibroblasts isolated from young or old donors, we have shown an increase in MMP1 and MMP3 levels in the culture medium in skin models constructed with old fibroblasts. These MMPs could contribute to the degradation of the human dermis matrix as described previously [20]. Indeed, MMP-1 is the major protease that cleaves and initiates fragmentation of collagen fibers like type I and III and thereafter the collagen can be degraded by MMP3 [21]. In addition to this degradation, previous studies have shown that collagen biosynthesis (for example, type I procollagen) was also reduced in fibroblasts that results in an apparent collagen deficiency during skin aging $[22,23]$. With the flax extract, the increase in MMPs was reduced and the expression of mRNA coding for collagens was increased in treated reconstructed skin.

During the aging process, other components of the extracellular matrix are found to be disorganized. The elastic network undergoes structural changes as seen for fibrillin-rich microfibrils in the papillary dermis, which are selectively degraded and disappear [24]. Also proteoglycans and glycosaminoglycans levels were reported to be reduced [25,26]. Dermal-epidermal junction (DEJ) was also affected with aging [27]. Langton, et al. reported a reduction in the protein distribution of several collagens type and laminin-332 in intrinsically aged skin [28]. This alteration of DEJ leads to a defect of epidermal renewal and homeostasis and may alter the intercellular communication between the dermis and the epidermis.

The communication between the dermis and the epidermis also depends on the stimulation of the keratinocytes by the fibroblasts of the papillary dermis. Thus, the synthesis of KGF by fibroblasts plays an important role in the maintenance of epidermal homeostasis [29].

Overall, it is interesting to note that the flax extract improved the expression of most of these biological actors of homeostasis that are altered during aging.

In addition to the biological properties of the skin that are altered during aging, mechanical properties are also altered. It has been described in vivo that mechanical properties such as elasticity decrease with age [30]. There is a close relationship between physical properties, cell biology and quality of skin components. Indeed, it has been shown that the fibroblast is reduced in size and collapses in a degraded matrix environment on which it can no longer fix (attach ?) and stretch $[20,31,32]$. This process facilitates the appearance of ROS, which in turn stimulate the production of MMPs $[33,34]$ and consequently degrades the matrix environment of the skin. The quality of the ECM is important to maintain the mechanical properties of the skin. It has been shown that the neosynthesis of collagen and elastin for example can improve the elasticity of the skin [35]. In our three-dimensional system, it is interesting to observe the improvement of the elastic parameters after treatment with the flax extract, which can be explained, in part, by the increase of ECM synthesis (as seen for procollagen 1 and fibrillin 1 ) and the decrease of its 
degradation by reducing the levels of the metalloproteinases MMP1 and MMP3.

It was difficult to attribute a specific activity of the flax extract to one of these components. Various studies carried out to analyze the composition of flax extracts were not consistent and depend on the extraction method used. However, results showed that the presence of compounds such as flavonoids, tannins, phenols or even tocopherol or $\beta$-carotenes in the extract. Among these compounds, some are known, like flavonoids or tocopherol, which reinforce the extracellular matrix component or stimulate the fibroblasts bioactivity [36] $\beta$-carotene inhibits metalloproteinase expression [37]. More recently, a global antioxidant and anti-aging effect has been described for flax [38]. The mechanisms of action of these different compounds are not necessarily well identified and their association could modulate their effects, but the result is an interesting overall activity for improving the homeostasis of the skin.

In conclusion, we have shown in vitro that flax extract could be a promising compound as an anti-aging strategy of the dermis. It appears to prevent or treat the effects of the skin aging by promoting the biosynthesis of the extracellular matrix and by reducing its degradation that improves both biological and mechanical properties.

\section{Acknowledgment}

We would like to thank Miss Girardeau-Hubert and Dr Mark Donovan for critical reading of this manuscript.

\section{References}

1. Zouboulis CC, Makrantonaki E. Clinical aspects and molecular diagnostics of skin aging. Clin Dermatol. 2011; 29: 3-14.

PubMed: https://pubmed.ncbi.nlm.nih.gov/21146726/

2. Kanaki T, Makrantonaki E, Zouboulis C. Biomarkers of skin aging. Rev Endocr Metab Disord. 2016; 17: 433-442.

PubMed: https://pubmed.ncbi.nlm.nih.gov/27830493/

3. Weber L, Kirsch E, Muller P, Krieg T. Collagen type distribution and macromolecular organization of connective tissue in different layers of human skin. J Invest Dermatol. 1984; 82: 156-160.

PubMed: https://pubmed.ncbi.nlm.nih.gov/6693779/

4. Zimmermann DR, Dours-Zimmermann MT, Schubert M, BrucknerTuderman,L. Versican is expressed in the proliferating zone in the epidermis and in association with the elastic network of the dermis. $J$ Cell Biol. 1994; 124: 817-825.

PubMed: https://pubmed.ncbi.nlm.nih.gov/8120102/

5. Sorrell JM, Caplan Al. Fibroblast heterogeneity: more than skin deep. J Cell Sci. 2004a; 117: 667-675.

PubMed: https://pubmed.ncbi.nlm.nih.gov/14754903/

6. Pageon H, Zucchi H, Asselineau D. Distinct and complementary roles of papillary and reticular fibroblasts in skin morphogenesis and homeostasis. Eur J Dermatol. 2012; 22: 324-332.

PubMed: https://pubmed.ncbi.nlm.nih.gov/22449755/

7. Mine S, Fortunel NO, Pageon $\mathrm{H}$, Asselineau D. Aging alters functionally human dermal papillary fibroblasts but not reticular fibroblasts: a new view of skin morphogenesisand aging. PLoS ONE. 2008; 3: e4066. PubMed: https://pubmed.ncbi.nlm.nih.gov/19115004/
8. Varani J, Warner RL, Gharaee-Kermani M, Phan SH, Kang S, et al. Vitamin A antagonizes decreased cell growth and elevated collagendegrading matrix metalloproteinases and stimulates collagen accumulation in naturally aged human skin. J Invest Dermatol. 2000; 114: 480-486.

PubMed: https://pubmed.ncbi.nlm.nih.gov/10692106/

9. Nusgens BV, Humbert P, Rougier A, Colige AC, Haftek M, et al. Topically applied vitamin C enhances the mRNA level of collagens I and III, their processing enzymes and tissue inhibitor of matrix metalloproteinase $\mathrm{I}$ in the human dermis. J Invest Dermatol. 2001; 116: 853-859. PubMed: https://pubmed.ncbi.nlm.nih.gov/11407971/

10. Asselineau D, Bernard BA, Bailly C, Darmon M. Retinoic acid improves epidermal morphogenesis. Dev Biol 1989; 133: 322-335. PubMed: https://pubmed.ncbi.nlm.nih.gov/2471653/

11. Phillips CL, Combs SB, Pinnell SR. Effects of ascorbic acid proliferation and collagen synthesis in relation to the donor age of human dermal fibroblasts. J Invest Dermatol. 1994; 103: 228-232.

PubMed: https://pubmed.ncbi.nlm.nih.gov/7518857/

12. Marionnet C, Vioux-Chagnoleau C, Pierrard C, Sok J, Asselineau D, et al. Morphogenesis of dermal epidermal junction in a model of reconstructed skin: beneficial effect of vitamin C. Exp Dermatol. 2006; 15: 625-633.

PubMed: https://pubmed.ncbi.nIm.nih.gov/16842601/

13. Pageon $\mathrm{H}$, Azouaoui A, Zucchi H, Ricois S, Tran C, et al. Potentially beneficial effects of rhamnose on skin ageing: an in vitro and in vivo study. Int J Cosmet Sci. 2019; 41: 213-220.

PubMed: https://pubmed.ncbi.nlm.nih.gov/30845349/

14. Rheinwald JG, Green H. Serial cultivation of strains of human epidermal keratinocytes: the formation of keratinizing colonies from single cells. Cell. 1975; 6: 331-343.

PubMed: https://pubmed.ncbi.nlm.nih.gov/1052771/

15. Asselineau D, Prunieras M. Reconstruction of simplified skin: control of fabrication. Br J Dermatol. 1984; 111: 219-222.

PubMed: https://pubmed.ncbi.nlm.nih.gov/6743553/

16. Asselineau D, Bernhard B, Bailly C, Darmon M. Epidermal morphogenesis and induction of the $67 \mathrm{kD}$ keratin polypeptide by culture of human keratinocytes at the liquid-air interface. Exp Cell Res. 1985; 159: 536-539.

PubMed: https://pubmed.ncbi.nlm.nih.gov/2411581/

17. Battie C, Verschoore M. Cutaneous solar ultraviolet exposure and clinical aspects of photodamage. Indian J Dermatol Venereol Leprol. 2012; 78/S9-S14.

PubMed: https://pubmed.ncbi.nlm.nih.gov/22710112/

18. Lago JC, Puzzi MB. The effect of aging in primary human dermal fibroblasts. PLoS One. 2019; 14: e0219165.

PubMed: https://pubmed.ncbi.nlm.nih.gov/31269075/

19. Verma RP, Hansch C. Matrix metalloproteinases (MMPs): Chemicalbiological functions and (Q)SARS. Bioorg Med Chem. 2007; 15: 22232268.

PubMed: https://pubmed.ncbi.nlm.nih.gov/17275314/

20. Quan T, Little E, Quan H, Qin Z, Voorhees JJ, et al. Elevated matrix metalloproteinases and collagen fragmentation in photodamaged human skin: impact of altered extracellular matrix microenvironment on dermal fibroblast function. J Invest Dermatol. 2013; 133: 1362-1366. PubMed: https://pubmed.ncbi.nlm.nih.gov/23466932/

21. Brennan M, Bhatti H, Nerusu KC, Bhagavathula N, Kang S, Fisher GJ, et al. Matrix metalloproteinase- 1 is the major collagenolytic enzyme responsible for collagen damage in uv-irradiated human skin. Photochem Photobiol. 2003; 78: 43-48.

PubMed: https://pubmed.ncbi.nlm.nih.gov/12929747/

22. Varani J, Dame MK, Rittie L, Fligiel SE, Kang S, et al. Decreased collagen production in chronologically aged skin: roles of agedependent alteration in fibroblast function and defective mechanical stimulation. Am J Pathol. 2006; 168: 1861-1868.

PubMed: https://pubmed.ncbi.nlm.nih.gov/16723701/ 
23. Quan C, Cho MK, Perry D, Quan T. Age-associated reduction of cell spreading induces mitochondrial DNA common deletion by oxidative stress in human skin dermal fibroblasts: implication for human skin connective tissue aging. J Biomed Sci. 2015; 22: 62.

PubMed: https://pubmed.ncbi.nlm.nih.gov/26215577/

24. Beylot C. Skin aging: clinico pathological features and mechanisms. Annales de Dermatologie. 2009; 136: S263-S269.

PubMed: https://pubmed.ncbi.nlm.nih.gov/19931682/

25. Oh JH, Kim YK, Jung JY, Shin JE, Chung JH. Changes in glycosaminoglycans and related proteoglycans in intrinsically aged human skin in vivo. Exp. Dermatol. 2011; 20: 454-456.

PubMed: https://pubmed.ncbi.nlm.nih.gov/21426414/

26. Lee $\mathrm{DH}$, Oh JH, Chung JH. Glycosaminoglycan and proteoglycan in skin aging. J Dermatol Sci. 2016; 83: 174-181.

PubMed: https://pubmed.ncbi.nlm.nih.gov/27378089/

27. Deloche C, Minondo AM, Bernard BA, Bernerd F, Salas F, et al. Effect of $\mathrm{C}$-xyloside on morphogenesis of the dermal epidermal junction in aged female skin. An ultrastuctural pilot study. Eur. J Dermatol. 2011; 21: 191-196.

PubMed: https://pubmed.ncbi.nlm.nih.gov/21454149/

28. Langton AK, Halai P, Griffiths CE, Sherratt MJ, Watson RE. The impact of intrinsic ageing on the protein composition of the dermal-epidermal junction. Mech Ageing Dev. 2016; 156:14-16.

PubMed: https://pubmed.ncbi.nlm.nih.gov/27013376/

29. Gibbs S, Silva Pinto AN, Murli S, Huber M, Hohl D, et al. Epidermal growth factor and keratinocyte growth factor differentially regulate epidermal migration, growth, and differentiation. Wound Repair Regen. 2000; 8: 192-203.

PubMed: https://pubmed.ncbi.nlm.nih.gov/10886810/

30. Krueger N, Luebberding S, Oltmer M, Streker M, Kerscher M. Agerelated changes in skin mechanical properties: a quantitative evaluation of 120 female subjects. Skin Res Technol. 2011; 17: 141-148. PubMed: https://pubmed.ncbi.nlm.nih.gov/21281361/

31. Fisher GJ, Quan T, Purohit T, Shao Y, Cho MK, et al. Collagen fragmentation promotes oxidative stress and elevates matrix metalloproteinase-1 in fibroblasts in aged human skin. Am J Pathol. 2009; 174: 101-114.

PubMed: https://pubmed.ncbi.nlm.nih.gov/19116368/

32. Fisher GJ, Shao Y, He T, Qin Z, Perry D, et al. Reduction of fibroblast size/mechanical force down-regulates TGF- $\beta$ type II receptor: implications for human skin aging. Aging Cell. 2016; 15: 67-76. PubMed: https://pubmed.ncbi.nlm.nih.gov/26780887/

33. Quan T, Fisher GJ. Role of Age-Associated Alterations of the Dermal Extracellular Matrix Microenvironment in Human Skin Aging: A MiniReview. Gerontology. 2015; 61: 427-434.

PubMed: https://pubmed.ncbi.nlm.nih.gov/25660807/

34. QinZ,BalimunkweRM, QuanT. Age-related reduction ofdermalfibroblast size upregulates multiple matrix metalloproteinases as observed in aged human skin in vivo. $\mathrm{Br} \mathrm{J}$ Dermatol. 2017; 177: 1337-1348. PubMed: https://pubmed.ncbi.nlm.nih.gov/28196296/

35. Yutskovskaya YA, Kogan EA. Improved Neocollagenesis and Skin Mechanical Properties After Injection of Diluted Calcium Hydroxylapatite in the Neck and Décolletage: A Pilot Study. J Drugs Dermatol. 2017; 16: 68-74.

PubMed: https://pubmed.ncbi.nlm.nih.gov/28095536/

36. Beroual K, Agabou A, Abdeldjelil MC, Boutaghane N, Haouam S, et al Evaluation of crude flaxeed (Linum usitatissimum $L$ ) oil in burn wound healing in new zealand rabbits. Afr J Tradit Complement Altern Med. 2017; 14: 280-286.

PubMed: https://pubmed.ncbi.nlm.nih.gov/28480439/

37. Stahl W, Sies H. $\beta$-Carotene and other carotenoids in protection from sunlight. Am J Clin Nutr. 2012; 96: 1179S-1184S. PubMed: https://pubmed.ncbi.nlm.nih.gov/23053552/

38. Bose $S$, Munsch T, Lanoue A, Garros L, Tungmunnithum D, et al. UPLC-HRMS Analysis Revealed the Differential Accumulation of Antioxidant and Anti-Aging Lignans and Neolignans in In Vitro Cultures of Linum usitatissimum L. Front Plant Sci. 2020; 11: 508658.

PubMed: https://pubmed.ncbi.nlm.nih.gov/33072140/ 